\title{
CROMOSOMAS DE ESPECIES AMERICANAS DE SIDA (MALVACEAE)
}

\author{
GRACIELA I. LAVIA ${ }^{1}$, AVELIANO FERNÁNDEZ1 \& ANTONIO KRAPOVICKAS ${ }^{1}$
}

\begin{abstract}
Summary: Lavia, G.I., A. Fernández \& A. Krapovickas. 2007. Chromosomes of American species of Sida (Malvaceae). Bonplandia 16(3-4): 255-258. ISSN: 0524-0476.

Chromosome numbers are reported for 26 accessions of Sida (Malvaceae) from Argentina, Bolivia, Brazil, México and Paraguay representing 15 species. First chromosome counts are cited for the following 8 species: S. Charpinii Krapov. $2 n=14$, S. ciliaris L. $2 n=16$, S. Monteiroi Krapov. 2n=16, S. anomala A. St.-Hil. 2n=16, S. Cristobaliana Krapov. 2n=32, S. dubia A. St.-Hil. \& Naudin 2n=14, S. Poeppigiana (K.Schum.) Fryxell 2n=14 and S. Leitaofilhoi Krapov. 2n=14.
\end{abstract}

Key words: Chromosome numbers, Sida, Malvaceae.

Resumen: Lavia, G.I., A. Fernández \& A. Krapovickas. 2007. Cromosomas de especies americanas de Sida (Malvaceae). Bonplandia 16(3-4): 255-258. ISSN: 0524-0476.

En este trabajo se presentan los números cromosómicos de 26 accesiones del género Sida (Malvaceae) de Argentina, Bolivia, Brasil, México y Paraguay, pertenecientes a 15 especies. Para las siguientes 8 especies se cita el número cromosómico por primera vez: S. Charpinii Krapov. $2 \mathrm{n}=14$, S. ciliaris L. $2 \mathrm{n}=16$, S. Monteiroi Krapov. $2 \mathrm{n}=16$, S. anomala A. St.-Hil. $2 \mathrm{n}=16$, S. Cristobaliana Krapov. 2n=32, S. dubia A. St.-Hil. \& Naudin 2n=14, S. Poeppigiana (K.Schum.) Fryxell 2n=14 y S. Leitaofilhoi Krapov. 2n=14.

Palabras clave: Números cromosómicos, Sida, Malvaceae.

\section{Introducción}

Los números cromosómicos constituyen información de fundamental importancia en estudios taxonómicos y evolutivos. Particularmente la familia Malvaceae, constituida por aproximadamente 110 géneros y 2300 especies, presenta algunos géneros que se diferencian por el número de cromosomas. Tal es el caso de Cienfuegosia Cav., que se distingue del resto de los géneros de la tribu Gossypieae por presentar $2 \mathrm{n}=20$ о 2n=22 (Fryxell, 1969).

El género Sida L. es pantropical con cerca de 120 especies, y además muy heterogéneo. Debi- do a la variabilidad tanto morfológica como cromosómica que presenta (Skovsted, 1935, 1941; Krapovickas, 1965; Bates, 1976; Fernández, 1974, 1981), fue subdividido en 12 secciones (Fryxell, 1985; Krapovickas, 2003). Citogenéticamente, algunas secciones se diferencian de otras por los números básicos, por ejemplo las especies de las secciones Malacroideae y Oligandrae presentan número básico $\mathrm{x}=8$, a diferencia de la mayoría de las secciones que tienen $x=7$ (Fryxell, 1997). Por lo tanto, la información cromosómica que se obtenga de las colecciones de Sida es relevante para la delimitación taxonómica de las mismas.

En este trabajo y como parte de los estudios

${ }^{1}$ Instituto de Botánica del Nordeste (UNNE-CONICET), Casilla de Correo 209, 3400 Corrientes, Argentina. E-mail: ibone@agr.unne.edu.ar 
citogenéticos que se desarrollan en Malvaceae, hemos determinado los números cromosómicos de 15 especies de Sida.

\section{Materiales y Métodos}

Los ejemplares testigo fueron identificados por uno de los autores (Ing. A. Krapovickas) y se encuentran depositados en el herbario del
IBONE (CTES). El material analizado se presenta en la Tabla 1.

Los recuentos cromosómicos se realizaron en ápices de raíces obtenidos de semillas germinadas en cajas de Petri, pretratadas en 8 oxiquinoleína $0,002 \mathrm{M}$, durante $3 \mathrm{~h}$ a temperatura de laboratorio, fijadas en 5 partes de etanol absoluto y una parte de ácido láctico (Fernández, 1974) y conservadas en alcohol $70^{\circ}$. La coloración se realizó siguiendo la técnica de Feulgen.

Tabla 1. Especies estudiadas, números cromosómicos, coleccionistas y localidades.

\begin{tabular}{|c|c|c|c|}
\hline Especie & $2 n$ & Coleccionista y localidad & Referencia \\
\hline S. angustissima A.St. Hil. & 14 & $\begin{array}{l}\text { K 45439. Paraguay, Boquerón, Ruta Transchaco, } 12 \\
\text { km NW de Nueva Asunción. }\end{array}$ & $\begin{array}{l}\text { Krapovickas } \\
\text { (1969) }\end{array}$ \\
\hline \multicolumn{4}{|l|}{ Secc. Cordifoliae } \\
\hline S. Charpinii Krapov. & 14 & $\begin{array}{l}\text { K 45403. Paraguay, Boquerón, Ruta Transchaco, } 8 \\
\text { km NW de Nueva Asunción. }\end{array}$ & * \\
\hline " & 14 & $\begin{array}{l}\text { K 45534. Paraguay, Boquerón, } 12 \text { km NW de } \\
\text { Nueva Asunción. }\end{array}$ & \\
\hline S. cordifolia L. & 28 & $\begin{array}{l}\text { K 45402. Paraguay, Boquerón, Ruta Transchaco, } 8 \\
\text { km NW de Nueva Asunción. }\end{array}$ & Skovsted (1941) \\
\hline S. variegata (Griseb.) Krapov. & 14 & $\begin{array}{l}\text { K 45451. Paraguay, Boquerón, Ruta Transchaco, } 7 \\
\text { km NW de Nueva Asunción. }\end{array}$ & $\begin{array}{l}\text { Krapovickas } \\
\text { (1957) }\end{array}$ \\
\hline \multicolumn{4}{|l|}{ Secc. Malacroideae } \\
\hline S. anomala A. St.-Hil. & 16 & K 7963. Argentina, Corrientes, Mercedes. & * \\
\hline S. ciliaris L. & 16 & K 45283. Paraguay, Fortín Tte. Ochoa. & * \\
\hline$"$ & 16 & SN 1065. Bolivia, 22 km E de S.Cruz. & \\
\hline " & 16 & SN 1410. Bolivia, Chuquisaca, Zudañez. & \\
\hline " & 16 & SN 1336. Bolivia, Sta. Cruz, Florida. & \\
\hline S. Cristobaliana Krapov. & 32 & $\begin{array}{l}\text { K 45401. Paraguay, Boquerón, Ruta Transchaco, } 8 \\
\text { km NW de Nueva Asunción. }\end{array}$ & * \\
\hline " & 32 & SN 1264, Bolivia, Sta. Cruz, S. Rafael. & \\
\hline S. Monteiroi Krapov. & 16 & K 47907. Argentina, Formosa, E. del Campo. & $*$ \\
\hline " & 16 & K 45255. Paraguay, Pte Hayes, Pozo Colorado. & \\
\hline$"$ & 16 & K 10599. Paraguay, Concepción. & \\
\hline$"$ & 16 & K 47766. Argentina, Salta, Los Blancos. & \\
\hline
\end{tabular}

Secc. Sida

S. dubia A. St.-Hil. \& Naudin

14 K 10603. Paraguay, Puerto Casado.

S. Leitaofilhoi Krapov.

14 K 33449. Brasil, Minas Gerais, Ouro Preto. 


\begin{tabular}{|c|c|c|c|}
\hline Especie & $2 n$ & Coleccionista y localidad & Referencia \\
\hline $\begin{array}{l}\text { S. lonchitis A St.-Hil. \& } \\
\text { Naudin }\end{array}$ & 14 & K 23243. Brasil, Rio de Janeiro, Petropolis. & Fernández (1981) \\
\hline$"$ & 14 & K 38444. Brasil, Santa Catarina, Porto Belo. & \\
\hline $\begin{array}{l}\text { S. Poeppigiana (K.Schum.) } \\
\text { Fryxell }\end{array}$ & 14 & K 4796. Argentina, Tucumán, San Javier. & $*$ \\
\hline " & 14 & K 46762. Bolivia, La Paz, Coroico. & \\
\hline S. rhombifolia L. & 14 & K 23598. Mexico, OA, Monte Albán. & Skovsted (1941) \\
\hline$"$ & 14 & D 646. Argentina, Misiones, San Javier, Ayo. Alipio. & \\
\hline S. tuberculata R.E.Fr. & 28 & K 46765. Bolivia, La Paz, Coroico. & Krapovickas (1957) \\
\hline
\end{tabular}

Abreviaturas de los coleccionistas: $\mathrm{K}=$ A. Krapovickas, $\mathrm{SN}=$ V. Solís Neffa, D = M. Dematteis. * recuento nuevo

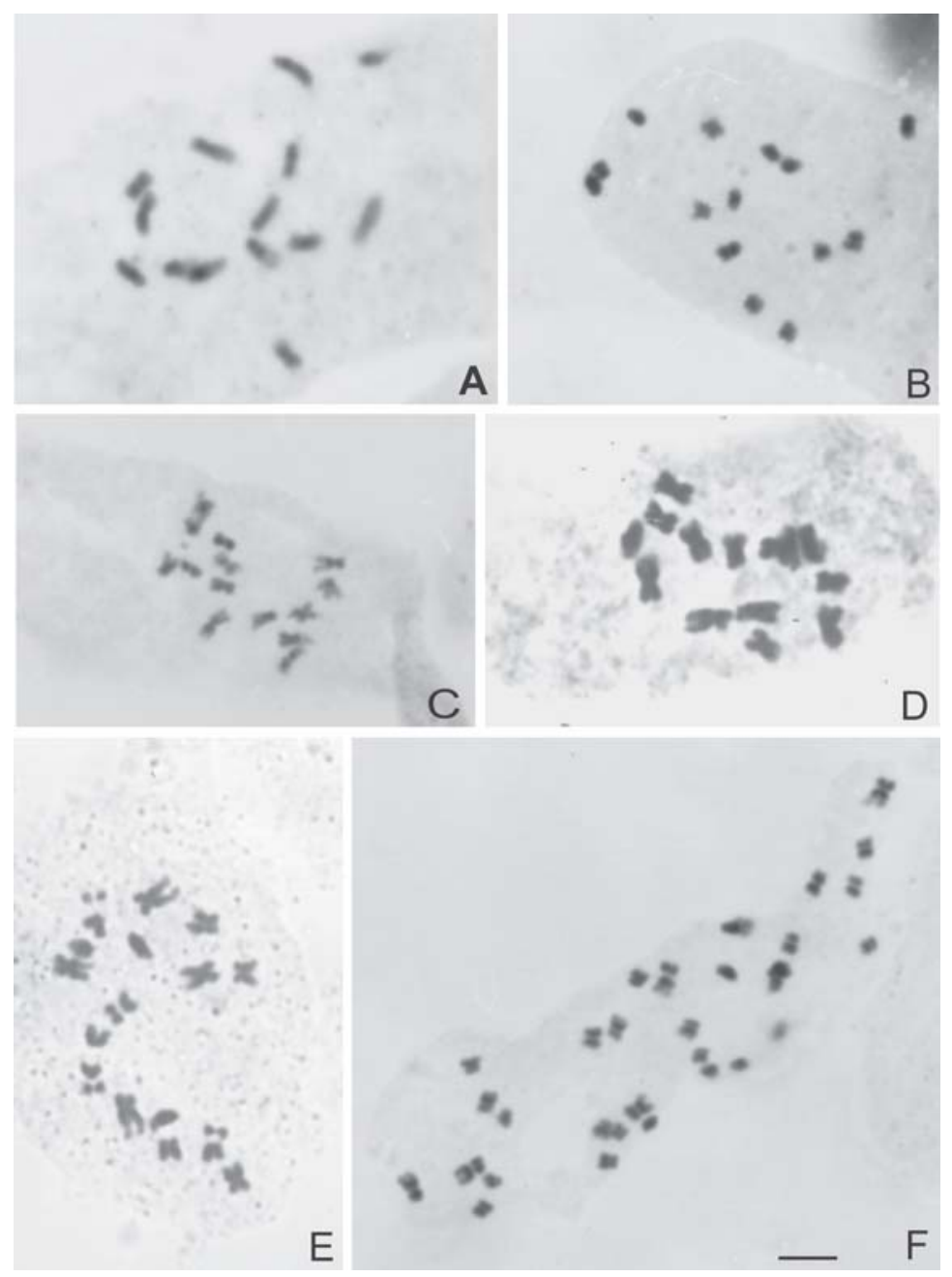

Fig. 1. Cromosomas mitóticos de especies de Sida. A: S. Charpinii 2n=14. B: S. Poeppigiana 2n=14. C: S. angustisssima 2n=14. D: S. variegata $2 \mathrm{n}=14$. E: S. ciliaris $2 \mathrm{n}=16$. F: S. Cristobaliana 2n=32. Barra= 5 micras. 


\section{Resultados y Discusión}

Los números cromosómicos hallados para las distintas especies estudiadas figuran en la Tabla 1. La mayoría de las especies de las secciones Cordifoliae y Sida resultaron diploides con $2 \mathrm{n}=14$, y dos tetraploides con 2n=28, siempre con número cromosómico básico $\mathrm{x}=7$. Para las especies $S$. Charpinii (Fig. 1A), S. dubia, S. Poeppigiana (Fig. 1B) y $S$. Leitaofilhoi, todas con $(2 n=14)$, este constituye el primer recuento. Por otra parte, se confirman los números cromosómicos de $S$. angustisssima (Krapovickas, 1969) (Fig. 1C), S. variegata (Krapovickas, 1957) (Fig. 1D), S. rhombifolia (Skovsted, 1941), S. lonchitis (Fernández, 1981) con 2n=2x=14, y con $2 \mathrm{n}=4 \mathrm{x}=28$ para S. cordifolia (Skovsted, 1941) y S. tuberculata (Krapovickas, 1957).

También se dan a conocer por primera vez los números cromosómicos para las 4 especies estudiadas de la sección Malacroideae, S. ciliaris (Fig. 1E), S. Monteiroi y S. anomala, diploides con $2 \mathrm{n}=16$ y $S$. Cristobaliana tetraploide con $2 \mathrm{n}=32$ (Fig. $1 \mathrm{~F}$ ), todas con número básico $\mathrm{x}=8$.

Con estos resultados se confirma el número básico único en las secciones analizadas, $\mathrm{x}=7$ para las secciones Cordifoliae y Sida y x=8 para Malacroideae.

\section{Bibliografía}

BATES, D.M. 1976. Chromosome numbers in the Malvales. III. Miscellaneous counts from the Byttneriaceae and Malvaceae. Gentes Herb. 11: 143-150.

FERNÁNDEZ, A. 1974. Recuentos cromosómicos en Malváceas. Bol. Soc. Argent. Bot. 15: 403-410.

—_. 1981 Recuentos cromosómicos en Malvales. Bonplandia 5 (10): 63-71.

FRYXELL, P.A. 1969. The genus Cienfuegosia Cav. (Malvaceae). Ann. Missouri Bot. Gard. 56: 179-250.

- 1985. Sidus sidarum V. The North and Central American species of Sida. Sida 11: 62-91.

- 1997. The American genera of Malvaceae. II. Brittonia 49: 204-269.

KRAPOVICKAS, A. 1957. Números cromosómicos de Malváceas americanas de la tribu Malveae. Revista Agron. Noroeste Argent. 2: 245-260.

—. 1965. Malvaceae. In A. L. Cabrera (Ed.), Flora de la Provincia de Buenos Aires. Colección Científica del INTA, Buenos Aires 4: 169-220.

—. 1969. Notas citotaxonómicas sobre Malváceas. Bonplandia 3: 9-24.

—_. 2003. Sida sección Distichifolia (Monteiro) Krapov. comb. nov., stat. nov. (MalvaceaeMalveae). Bonplandia 12: 83-122.

SKOVSTED, A. 1935. Chromosome numbers in the Malvaceae. I. I. J. Genet. 31: 262-296.

—. 1941. Chromosome numbers in the Malvaceae. II. C.R. Lab. Carlsberg Physiol. 23: 195-242.

Original recibido el 30 de septiembre de 2007; aceptado el 2 de noviembre de 2007. 